\title{
Topical immunization strategies
}

\author{
C Czerkinsky $^{1}$ and J Holmgren ${ }^{2}$
}

Research has yielded an abundance of vaccine candidates against mucosal infections, but only few mucosal vaccines have been registered for human use. Extensive research is being carried out to identify new and safe adjuvants for mucosal immunization, novel delivery systems, including live vectors and reporter molecules for tissue- and cell-specific targeting of vaccine antigens. If these candidates are to reach those in need, several lessons from clinical and field research carried out under resource-poor settings must be considered. These lessons include the need to develop new vaccines that can be administered topically onto the skin or to the mucosa, without needles or expensive delivery devices. Such topical vaccines must be able to protect all age groups at risk, be safe and effective in immunocompromised people, and be able to contain epidemics following complex emergencies. The anatomical compartmentalization of immune responses imposes constraints on the selection of topical route(s) of vaccine administration and on strategies for measuring these responses, especially in young infants. Thus, the selection of any particular route of immunization is critical when designing and formulating vaccines against organ-specific infections.

\section{INTRODUCTION}

Epithelial surfaces covering the skin (epidermis), aerodigestive mucosa, urogenital tract, the eye conjunctiva, inner ear, and the ducts of all exocrine glands are endowed with powerful mechanical and physicochemical cleansing mechanisms that repel and degrade most foreign matters. Although the dermoepidermal compartment of the skin is relatively poorly populated with effector immunocytes and the stratum corneum overlaying the epidermis is impermeable to most water-soluble macromolecules, the mucosa-associated lymphoid tissue (MALT) comprises an abundance of immune cells (nearly $80 \%$ of all immunocytes in a healthy human adult), commensurate with the size of surfaces it has to protect, and the inherent permeability of mucosal epithelia to a variety of foreign matters, including nutrients and products from commensal microbiota.

The MALT represents an example of highly compartmentalized immunological system that functions essentially independent from the systemic immune apparatus and from the skin-associated lymphoid tissue. Furthermore, and at variance with the systemic immune apparatus, which operates in a normally sterile milieu, the MALT guards organs that are replete with foreign antigens. Only a limited proportion of foreign antigens are derived from exposure to pathogens, and most are commensal microorganisms, food proteins, and other ingested or inhaled environmental matters. This implies that upon encounter with this broad range of antigenic stimuli, the MALT must economically select appropriate effector mechanisms and regulate their intensity to avoid bystander tissue damage.

The majority of microbial pathogens have a mucosal port of entry. Parenteral vaccination can provide protection in some instances, particularly in individuals previously exposed or vaccinated at a mucosal site, but in most cases, a mucosal route of vaccination is necessary for inducing a local immune response. In addition, compared with injectable vaccines, mucosal vaccines are easier to administer, carry less risk of transmitting infections, and could simplify manufacturing processes, thereby facilitating vaccine production and mass deployment.

In the early days of mucosal immunology, the concept of a "common mucosal immune system" whereby immune responses initiated at one mucosal site could disseminate to virtually any mucosal tissues led to the widely held notion that immunization by any mucosal route, such as oral vaccination, could be used for inducing effective immune responses not only in the gastrointestinal tract, but also in the airways and the urogenital tract. However, increasing evidence has shown that while this concept is by and large valid, mucosal immune responses exhibit a fair degree of compartmentalization, not only between separate mucosal organs ${ }^{1-4}$ but also between regions from the same mucosal organ, such as the gut, an observation reported more than 40 years ago by Ogra and Karzon. ${ }^{4}$

In this review, we summarize others and our own studies concerning the anatomical distribution of immune responses after

${ }^{1}$ International Vaccine Institute, Seoul, South Korea. ${ }^{2}$ Institute of Biomedicine, Department of Medical Microbiology and Immunology, Sahlgrenska Academy at Gothenburg University, Göteborg, Sweden. Correspondence: C Czerkinsky (cczerkinsky@ivi.int) or J Holmgren (jan.holmgren@microbio.gu.se) 
Table 1 Different types of mucosal infections may call for different types of vaccines

\begin{tabular}{|c|c|c|c|}
\hline Type of infection & Pathogens & $\begin{array}{l}\text { Type of vaccine } \\
\text { needed }\end{array}$ & $\begin{array}{l}\text { Examples of vaccines (licensed or at an } \\
\text { advanced stage of development) }\end{array}$ \\
\hline \multirow[t]{2}{*}{ Noninvasive and noninflammatory } & V. cholerae & Mucosal & $\begin{array}{l}\text { Killed cholera bacteria } \pm \text { B subunit } \\
\text { Live-attenuated cholera bacteria }\end{array}$ \\
\hline & $\begin{array}{l}\text { Enterotoxigenic } \\
\text { E. coli (ETEC) }\end{array}$ & & Transcutaneous ETEC LT vaccine \\
\hline $\begin{array}{l}\text { Invasive, inflammatory, and } \\
\text { systemic spread }\end{array}$ & $\begin{array}{l}\text { S. typhi } \\
\text { S. pneumoniae } \\
\text { H. influenzae type B } \\
\text { Polio virus } \\
\text { Influenza virus } \\
\text { HIV } \\
\text { M. tuberculosis }\end{array}$ & $\begin{array}{l}\text { Mucosal and/or } \\
\text { parenteral }\end{array}$ & $\begin{array}{l}\text { Injectable Vi-based typhoid vaccines (Vi polysaccharide } \\
\text { and Vi-protein conjugate vaccines) } \\
\text { Oral live-attenuated typhoid vaccine } \\
\text { Oral BCG (Moreau RDJ) } \\
\text { Injectable S. pneumoniae PS conjugate vaccines } \\
\text { Injectable HIB conjugate vaccines } \\
\text { Oral and injectable polio vaccines } \\
\text { Nasal live-attenuated influenza vaccines } \\
\text { Killed influenza whole and split virus vaccines }\end{array}$ \\
\hline Noninvasive and inflammatory & HPV & $\begin{array}{l}\text { Mucosal and/or } \\
\text { parenteral }\end{array}$ & Injectable HPV vaccines \\
\hline
\end{tabular}

Abbreviations: BCG, Bacillus Calmette-Guérin vaccine; ETEC, enterotoxigenic Escherichia coli; HIB, Haemophilus influenzae type B vaccine; HIV, human immunodeficiency virus; HPV, human papillomavirus; LT, E. coli heat-labile toxin.

topical immunization by different mucosal routes. We will also discuss the potential of sublingual and transcutaneous immunization, which have obtained promising results in preclinical and early clinical studies.

\section{MUCOSAL VACCINES: AN UNMET NEED}

Most infections are caused by pathogens which have a mucosal portal of entry, hence the need to develop mucosal vaccines against the many respiratory and enteric infections, especially during early childhood. It is estimated that mucosal respiratory and gastrointestinal infections each year cause more than ten billion disease episodes and kill approximately five million children under the age of 5 years, mainly in developing countries. To date, only a few mucosal vaccines have been licensed for human use, compared with the more than 30 injectable registered vaccines. All registered mucosal vaccines are for oral use against enteric infections except for two cold-adapted attennuaed nasal influenza vaccines (Table $\mathbf{1}$ ).

A large number of pathogens cause or start infections in the gastrointestinal tract (e.g., Helicobacter pylori, Vibrio cholerae, enterotoxigenic Escherichia coli (ETEC), Shigella spp., Salmonella spp., Clostridium difficile, polioviruses, rotaviruses, and noroviruses). Several other pathogens instead cause acute or chronic respiratory infections (e.g., group A streptococci, Streptococcus pneumoniae, Haemophilus influenzae, Mycoplasma pneumoniae, Mycobacterium tuberculosis, influenza virus, parainfluenza viruses, and respiratory syncytial virus). There are also a number of sexually transmitted mucosal pathogens (e.g., human immunodeficiency virus, human papillomavirus (HPV), Chlamydia, Neisseria gonorrhoeae, and herpes simplex virus). Collectively, infections caused by these agents represent an enormous challenge for developing vaccines that can induce protection against and interfere with person-to-person transmission of pathogens.

It is highly probable that infection by and interperson transmission of most mucosal pathogens can be effectively controlled by vaccination, provided vaccines are rationally designed and appropriately formulated to be administered by a given mucosal route. However, the nature of the pathogen and of the target mucosal tissue(s) will determine whether the vaccine should be given mucosally or alternatively parenterally in order to be efficacious (Table 2). A topical-mucosal route of vaccination seems to be critical for protecting against noninvasive infections at mucosal surfaces. Such infections involve pathogens that remain on the apical (luminal) side of mucosal epithelia, i.e., at sites that are (i) poorly accessible to antibodies transudating from blood, and (ii) where such blood-derived monomeric immunoglobulins (IgG, IgA) are insufficiently concentrated on the apical cell surface (due to lack of or limited receptor-mediated transport) and/or are unstable to function in the mucosal external environment. $V$. cholerae and ETEC infections are typical examples of such infections, in which vaccine-induced protection appears to be mediated mainly, if not exclusively, by locally produced secretory Ig (S-Ig) antibodies, and is associated with immunological memory.

On the other hand, when an infection occurs in the respiratory and urogenital mucosae, which are more permeable than the intestines to transudation by plasma antibodies, a parenteral route of vaccination may also be effective. The same may hold true for enteric infections in which the pathogen is first translocated across the epithelial barrier by intestinal $\mathrm{M}$ cells and then infects the basolateral side of the epithelium, as is the case with Shigella spp., or when the pathogen causes disease only after multiplying and producing inflammation in the submucosal 
Table 2 Comparative anatomical dissemination of secretory immunoglobulin A antibody responses after different routes of immunization

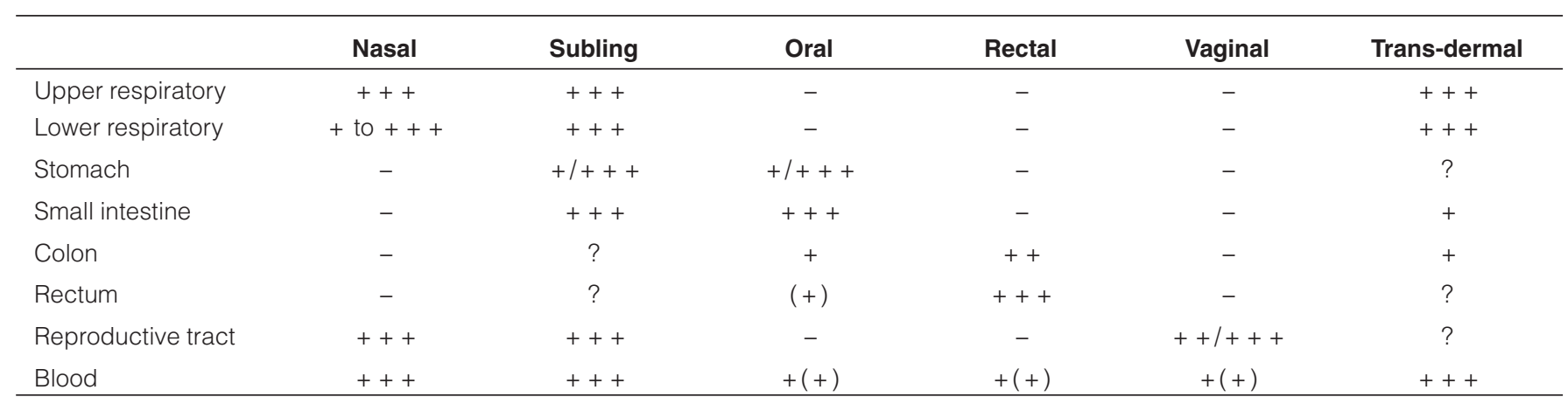

tissues, as is the case for infections caused by Campylobacter and most Salmonella bacteria. Finally, parenteral vaccines are clearly efficacious for many viral and bacterial infections when the pathogen has a mucosal portal of entry but then enters the blood for further systemic spread. Typical pathogens in this category for which effective injectable vaccines exist include S. pneumoniae, $H$. influenzae, S. typhi, poliovirus, and influenza virus. It is notable that for the last three indications, a mucosal vaccine is also available.

Taken together, and amid knowledge gaps in our understanding of the mucosal immune system and in our ability to measure its effector and memory arms, these considerations highlight the challenges faced by vaccinologists when attempting to design and formulate mucosal vaccines.

\section{CELLULAR AND MOLECULAR BASIS OF COMPARTMENTALIZED MUCOSAL IMMUNE RESPONSES}

The MALT is comprised of anatomically defined lymphoid microcompartments, which serve as the principal mucosal inductive sites where antigens are being ferried and where immune responses are initiated. ${ }^{1,5,6}$ Examples of such inductive sites are the Peyer's patches in the small intestine (mainly in the ileum); the abundant lymphoid follicles in the appendix, colon, and rectum; the mesenteric lymph nodes; the tonsils and adenoids at the entrance of the aerodigestive tract; and the many mucosal lymphoid follicles interspersed within the nasal mucosa and the bronchi of the respiratory tract mucosa (although it seems that the latter structures are less prominent in humans compared with some animal species). The MALT also contains a diffuse accumulation of a large number of lymphoid cells in the parenchyma of mucosal organs and exocrine glands, which represent the mucosal effector sites where immune responses are expressed.

Consistent with its high degree of compartmentalization, the MALT is populated by phenotypically and functionally distinct B-, T-, and accessory cell subpopulations, ${ }^{1}$ and has also developed strong restrictions upon lymphoid cell recirculation between mucosal sites.

Antigens may either penetrate or be taken up in mucosal inductive sites through a variety of mechanisms. One such example is the gut where the presence of a mucosal lymphoid follicle influences the adjacent intestinal epithelium by inducing differentiation of the so-called $\mathrm{M}$ cells. ${ }^{7}$ The latter cells, which cover the dome of intestinal Peyer's patches, have special properties for transporting antigens across the epithelium. ${ }^{8}$ Aside from other mechanisms of paracellular and transcellular uptake of macromolecules and particles through epithelia, an additional "capture" mechanism has been proposed and involves dendritic cells (DCs) protruding antigen-sampling dendrites across the intestinal epithelium. ${ }^{9}$

Irrespective of sampling mechanism, antigens taken up at a mucosal surface can then be ferried to, or directly captured by professional antigen-presenting cells (APCs), and presented to conventional CD $4+$ and CD8 $+\alpha \beta$ T cells. Certain antigens may also be processed and presented directly by epithelial cells to neighboring intraepithelial T cells, including $\mathrm{T}$ cells with limited repertoire diversity $(\gamma \delta$ T cells and natural killer T cells). With the majority of antigens, this results in suppression of specific immunity or "oral tolerance". ${ }^{10}$ However, an active immune response may also ensue, depending on the nature of the antigen, the type of APCs involved, and the characteristics of the local microenvironment. In general, inflammatory conditions favor the development of productive immune responses, and these immune responses are triggered by pathogens harboring motifs sensed by mucosal APCs as "danger signals" (e.g., Toll-like receptor ligands). ${ }^{11,12}$ Such stimulation of the mucosal innate immune system is an important reason why pathogens, and often live-attenuated bacterial or viral vaccines and killed bacterial whole-cell vaccines induce an immune response rather than tolerance. Selected subunit vaccines may also induce strong immune response by possessing similar or functionally analogous motifs, but most subunit vaccines, which essentially include parenteral subunit vaccines, need to be delivered with a proinflammatory adjuvant to stimulate a strong immune response. In most cases, a mucosal immune response appears to critically depend on appropriate antigen presentation by subsets of immunostimulatory mucosal DCs, except when very large amounts of mucosal antigens can trigger a mucosal IgA response in animals depleted of DCs. ${ }^{13}$ 
Sensitized mucosal immunocytes, both $\mathrm{T}$ and $\mathrm{B}$ cells, including IgA plasma cell precursors, leave the site of initial antigen encounter (e.g., in the Peyer's patch), transit through the lymph, enter the blood circulation, and then seed selected mucosal sites, preferentially the mucosa of origin but also remote mucosal tissues (such as the mammary and salivary glands after orogastric immunization, or the reproductive tract mucosa after nasal or sublingual immunization). During their migration to their final tissue location, mucosal lymphocytes differentiate into memory or effector cells. The anatomic affinity of such cells appears to be largely determined through interactions between site-specific integrins ("homing receptors") and chemokine receptors on mucosal lymphoid cells, and complementary tissuespecific endothelial cell adhesion molecules ("addressins") and chemokines, which are expressed differentially in the various mucosal tissues. ${ }^{14,15}$ This explains why mucosal lymphocytes preferentially traffic to mucosal as opposed to peripheral organs and tissues, and also why such cells may exhibit different affinity for distinct mucosal tissues. For instance, gut-homing IgA $\mathrm{B}$-cell precursors and their plasmablast progenitors, as well as mucosal memory T cells, express $\alpha 4 \beta 7$ integrin that can specifically attach to vascular MadCAM-1, a tissue-specific addressin that is expressed selectively on the endothelium of the gastrointestinal tract. ${ }^{15}$

Mucosal DCs in concert with neighboring epithelial cells have a critical role in this process by programming $\mathrm{T}^{16-19}$ and $\mathrm{B}$ cells ${ }^{20}$ to express specific homing receptors and by determining their fates and hence the nature of ensuing T- (helper, inflammatory, regulatory, cytotoxic) and B- (IgA, IgM, IgG, IgE) cell responses. ${ }^{9,12}$ Likewise, chemokines produced in the local epithelial microenvironment promote chemotaxis of immune cells with cognate chemokine receptors. ${ }^{9,21}$ For instance, in the gastrointestinal tract, CCL-28, also known as mucosa-associated epithelial chemokine, selectively attracts IgA B cells and plasmablasts expressing the chemokine receptor CCR-10, whereas CCL-25 (TECK), which is abundantly produced in the small intestine, can selectively attract $\mathrm{B}$ and $\mathrm{T}$ cells expressing the CCR-9 receptor to preferentially disseminate from the blood into the small intestinal mucosa. ${ }^{21}$ These types of specific molecular interaction between homing receptor molecules and chemokine receptors selectively "imprinted" at the mucosal inductive sites on the one hand, and addressins and chemokines selectively produced in the target mucosal tissues on the other hand, may explain both the segregation of secretory immune responses from systemic immune responses, and the preferential dissemination of mucosal responses to privileged mucosal sites.

\section{Preferential dissemination of mucosal immune responses after different routes of vaccination}

The described compartmentalization within the mucosal immune system places constraints on the choice of vaccination route for inducing effective immune responses at the desired sites. Administration of antigens by rectal, vaginal, and more recently, sublingual routes has been explored but only for experimental purposes so far, and mostly to study S-IgA antibody responses. In general, the strongest immune response is usually obtained at the site of initial vaccine exposure and in anatomically adjacent regions. However, a few notable exceptions have been found that may allow for more practical vaccine administration than would otherwise be possible, especially against infections in the urogenital tract. This has obviously practical implications for the development of mucosal vaccines against sexually transmitted and urogenital infections.

Traditional routes of mucosal immunization include the oral and nasal routes. When antigens with inherent mucosal immunogenicity and adjuvanticity are used, as is the case with cholera toxin (CT) and its B subunit (CTB), oral immunization may induce a substantial antibody response in mainly the small intestine (and then strongest in the proximal segment), in the ascending colon (Figure 1), the stomach, and in the mammary and salivary glands $\mathrm{s}^{22-27}$ (Table 1). Oral immunization, however, is relatively inefficient at evoking an S-IgA antibody response in the distal segments of the large intestines (Figure 1), the tonsils, the lower airway mucosa, or the reproductive tract mucosa. ${ }^{23-29}$ Conversely, rectal immunization evokes a strong local antibody response in both the rectum and sigmoid colon, and, although weaker, also in the descending colon, but little, if any response, in the small intestine and the proximal colon ${ }^{23,24}$ (Figure 1). On the other hand, nasal or tonsillar immunization in humans results in antibody responses in the upper airway mucosa and regional secretions (saliva, nasal secretions) ${ }^{30}$ without evoking an immune response in the gut. ${ }^{31,32}$ Local topical vaginal immunization also induces a local response in the genital secretions and in serum. ${ }^{28,29,33}$ It should be pointed out that although the latter studies were conducted with exceptionally potent immunogens, it is likely that the nature of the antigen/immunogen, adjuvant, and/or delivery system employed will have a profound influence on the intensity and type(s) of ensuing immune responses.

However, of particular interest for vaccination against sexually transmitted infections, nasal immunization has been found to give rise to substantial IgA and IgG antibody responses in the human cervicovaginal mucosae. ${ }^{33,34}$ The magnitude of the response achieved in the genital mucosa of women after intranasal immunization with CTB appears to be fully comparable to that seen when the vaccine is given by topical vaginal application. ${ }^{33,34}$

Apart from differences in the anatomical dissemination of S-IgA antibody responses induced by orogastric and intranasal immunization, respectively, the kinetics of the responses also appears to be markedly different. Several studies have shown that the intestinal antibody response after oral immunization is rapid and relatively short-lasting, but it is associated with a very long-lasting immunological memory. After oral cholera vaccination, data from extensive field trials have shown that although protection from the acute intestinal IgA response appears to vanish after 6-9 nine months, overall protection lasts for several years, which is consistent with the demonstration of mucosal immunological memory lasting for at least 5 years in Swedish volunteers after oral cholera vaccination. ${ }^{35}$ Rudin et al. ${ }^{33}$ compared the kinetics and organ distribution of the 


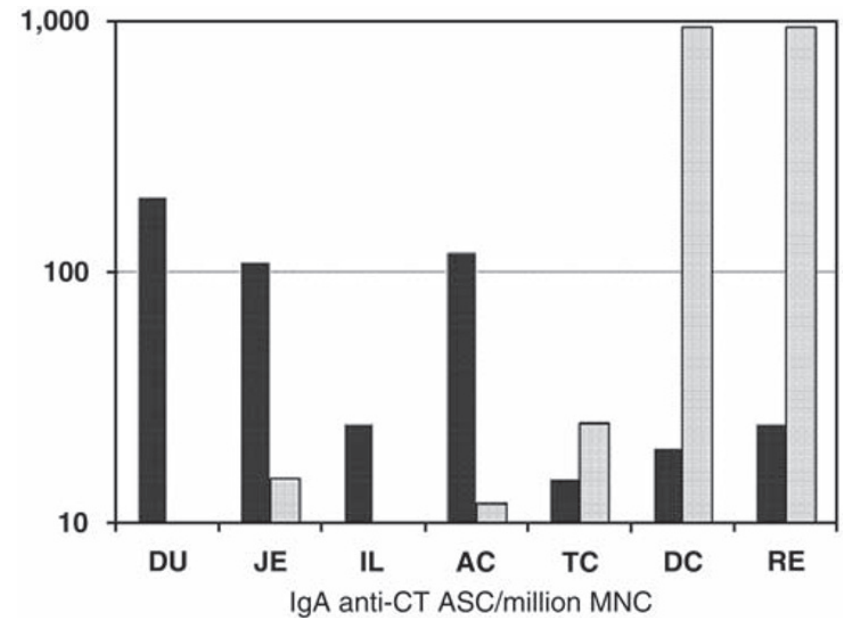

Figure 1 Compartmentalization of intestinal antibody responses after orogastric and rectal administration of cholera toxin (CT) in macaques. Data represent mean numbers of CT-specific IgA antibody-secreting cells detected in macaques after two immunizations with $\mathrm{CT}$ given by the orogastric (dark bars) or the rectal (clear bars) routes. AC, ascending colon; ASC, antibody-secreting cell; DC, descending colon; DU, duodenum; IL, ileum; JE, jejunum; MNC, mononuclear cell; RE, rectum; $\mathrm{TC}$, transverse colon. Adapted from Eriksson et al. ${ }^{23}$

antibody response after nasal and oral vaccination. They immunized Swedish female volunteers nasally or orally with CTB and determined the specific antibody levels in serum, and in nasal and vaginal secretions before immunization and at different times thereafter. Nasal vaccination induced strong increases in CTB-specific IgA and IgG antibodies in nasal secretions, whereas no significant nasal IgA response was seen after oral vaccination. Strong serum antibody responses to CTB were induced by both routes of vaccination. A striking difference between nasal and oral vaccination was that the nasal route elicited a specific antibody response with a later onset but of much longer duration than the oral route in both serum and at the mucosal expression sites.

The above considerations clearly indicate that the route of administration of vaccines has a profound effect on the characteristics and tissue distribution of immune responses, and have prompted efforts to explore alternative routes of vaccine delivery. In this respect, the potential of the sublingual ("under the tongue") route for administering vaccines is gaining increased interest because of recent studies indicating that this route may favor induction of broadly disseminated mucosal and systemic immune responses (Table 3). Over the past few years, we and others have shown that sublingual administration of a variety of soluble, as well as particulate antigens, including live and killed bacteria and viruses, can evoke a broad spectrum of immune responses in mucosal and extramucosal tissues, ranging from secretory and systemic antibody responses to mucosal and systemic cytotoxic T-cell responses. ${ }^{36}$ Although only studied in animals so far, in all instances where this route of administration has been compared with the classical orogastric route, sublingually induced responses have been far more pronounced and required 10- to 50-fold lower amounts of antigen for inducing S-IgA and serum antibody responses. ${ }^{37}$ Moreover, and contrary
Table 3 Sublingual immunization induces broadly disseminated protective immune responses in mice

\begin{tabular}{lclc}
\hline & $\begin{array}{c}\text { Serum IgG } \\
\text { Ab titer, } \\
\text { log10 }\end{array}$ & $\begin{array}{c}\text { Mucosal IgA } \\
\text { Ab titer, log10 }\end{array}$ & $\begin{array}{c}\text { Protection } \\
\text { against } \\
\text { infection, \% }\end{array}$ \\
\hline $\begin{array}{l}\text { Respiratory } \\
\text { (Influenza H1N1) }\end{array}$ & 4.0 & 2.7 (BAL fluid) & 100 \\
$\begin{array}{l}\text { Gastrointestinal } \\
\text { (H. pylori) }\end{array}$ & 4.7 & $\begin{array}{l}2.6 \text { (stomach } \\
\text { mucosa) }\end{array}$ & 95 \\
Genital (HPV) & 4.7 & $\begin{array}{l}2.5 \text { (vaginal } \\
\text { wash) }\end{array}$ & 100 \\
\hline
\end{tabular}

Abbreviations: Ab, antibody; BAL, broncho-alveolar lavage; HPV, human papillomavirus.

Adapted from Song et al., ${ }^{37}$ Raghavan et al. (to be published), and Cuburu et al. ${ }^{38}$

to oral (orogastric) immunization, sublingual administration of killed, as well as live-attenuated, influenza vaccine induced anti-viral responses in the lungs of mice, and protected mice against lethal respiratory challenge with infectious virions. ${ }^{37}$ More importantly, antigens and adjuvants that have been administered sublingually do not appear redirected to the olfactory bulb epithelium; thus sublingual vaccines are less likely to have the same safety issues as intranasal vaccines. More recently, we have documented that similar to intranasal immunization but at variance with orogastric immunization, sublingual administration of nonreplicating antigens can also induce secretory antibody responses, and, depending on the adjuvant used, cytotoxic T-cell response in the female reproductive tract mucosa. ${ }^{38}$ Another significant finding is that sublingual administration of a vaccine consisting of virus-like particles (VLPs) from HPV, given without an adjuvant, evoked virus-neutralizing antibody responses in both serum and genital secretions, and provided protection against genital challenge with HPV. ${ }^{38}$ Other recent experiments have shown that sublingual administration of an experimental $H$. pylori vaccine can effectively induce B- and Tcell responses in the stomach mucosa and protect mice against infection with $H$. pylori with an efficacy exceeding that achieved by orogastric immunization..$^{39}$ Finally, sublingual immunization with experimental ETEC and $V$. cholerae whole-cell vaccines, as well as purified fimbrial antigens, has proven to be efficient for inducing strong $\operatorname{IgA}$ antibody responses in the intestine, suggesting that this route may even be an alternative to the oral route for vaccinating against enteric infections ( $\mathrm{J}$ Holmgren and C Czerkinskey, unpublished data).

The exceptional ability of the murine sublingual mucosa to disseminate effector $\mathrm{B}$ - and $\mathrm{T}$-cell responses to various mucosal tissues appears to be contributed by specialized DCs residing in the sublingual epithelial and draining (submaxillary cervical) lymph nodes. These CCR7 + DCs appear to respond to the chemokines CCL19 and CCL2 $1^{40}$ produced in the local microenvironment and may imprint $\mathrm{B}$ and $\mathrm{T}$ cells (including possibly cytotoxic T-cell precursors) in draining cervical lymph nodes to migrate to CCL28-the mucosa-associated epithelial chemokine-which is expressed by columnar epithelial cells in the gut, lung, breast, and the salivary glands, and drives the mucosal homing of $\mathrm{T}$ and $\mathrm{B}$ lymphocytes that express CCR $10 .{ }^{41-43}$ 
Controlled clinical trials are now being conducted to determine the safety, tolerability, and efficiency of this novel route of administration. The development of suitable adjuvants and that of mucoadhesive formulations with enhanced permeabilizing properties to facilitate and prolong contact of vaccine antigens with the sublingual epithelium are likely to have major impact on the future emergence of sublingual vaccines.

Another interesting route of vaccine administration relates to the use of skin-adhesive patches containing antigens and adjuvants. ${ }^{44}$ This approach, also called "transcutaneous immunization," has been shown to induce both systemic, intestinal, and respiratory antibody responses in mice coadministered protein antigens together with CT or E. coli heat-labile enterotoxin (LT). Results of a clinical trial involving administration of LT as a stand-alone travelers' diarrhea vaccine appear to be promising, and suggest that transcutaneous immunization with potent adjuvant-active antigens, such as LT, may also evoke both intestinal and systemic antibody responses in humans. ${ }^{45}$ The ability of transcutaneous immunization to elicit intestinal antibody responses is intriguing and may relate to the observation that transcutaneously applied antigens coadministered with CT/LTlike adjuvants may induce expansion of bone marrow-derived DCs in mesenteric lymph nodes, which could promote isotype switching and differentiation of precursors of IgA antibodysecreting cells expressing CCR9 and CCR10 that can migrate to the intestines. ${ }^{46}$ The use of an adjuvant (immunostimulant) patch to condition the skin before injection of vaccines has given interesting results in clinical studies. ${ }^{45}$ This approach could be used for dose-sparing purposes with vaccines in short supply (e.g., pandemic influenza vaccines) or to enhance immune responsiveness to vaccines in the elderly.

Finally, as discussed above (see also Table 1), parenteral vaccination may in itself be useful for immunization against those mucosal infections in which the pathogen is taken up or penetrates across the epithelium. For instance, parenteral vaccineinduced serum IgG antibodies can protect against intestinal pathogens either by preventing subepithelial microbial spread (e.g., shigellosis) or invasion through draining vessels (e.g., typhoid). In addition, parenteral administration might be used in tandem with mucosal vaccines, whether the latter are given by oral, nasal, or sublingual route. Parenteral polio or cholera vaccines given as a booster have been found to stimulate antigen-specific S-IgA responses in naturally primed individuals, although they did not induce any such response when given to immunologically naïve individuals. ${ }^{47,48}$ Thus, injectable and mucosal vaccines might synergize with each other in their protective profiles if given in tandem.

\section{TOPICAL DELIVERY SYSTEMS AND ADJUVANTS}

The development of topically administered vaccines, whether for mucosal or transcutaneous administration, requires efficient antigen delivery and adjuvant systems. Ideally, these systems should protect the vaccine from physical elimination and enzymatic digestion, facilitate antigen uptake across the epithelium and target subepithelial APCs, and usually also appropriately stimulate the innate immune system to generate effective adaptive immunity. The latter effect is probably most critical for rendering subunit vaccines sufficiently immunogenic, and is a function expected to be provided by coformulated adjuvants to overcome the lack of normally intrinsic adjuvant activity of most live or killed whole-microbe vaccines. Although there is no mucosal vaccine containing a specific coformulated adjuvant or delivery system yet licensed for human use, there is now a wide range of promising approaches that give realistic hope for the future-these are summarized below and in several recent reviews. ${ }^{3,49-51}$

\section{Mucosal delivery systems}

A multitude of such systems have been developed, including both various inert systems and different live-attenuated bacterial or viral vector systems.

Among the inert systems are lipid-based structures with entrapped antigens such as liposomes, ${ }^{52}$ immune-stimulating complexes (ISCOMs), ${ }^{53}$ and cochleates; $;{ }^{54}$ different biodegradable particles based on, e.g., starch, ${ }^{55}$ poly(DL-lactide-co-glycolide) microspheres; ${ }^{56}$ and polymers of phosphazene and especially micron-sized hydrogel microspheres of crosslinked polyphosphazene strands. ${ }^{57,58}$ In addition, various proteins with binding affinity for epithelial surfaces have been reported to enhance antigen uptake, including both classical plant lectins and bacterial proteins such as CTB or LTB, to which antigens have been linked either chemically or through gene fusion technology.

Two main categories of live bacterial vectors can be distinguished, one based on attenuated pathogens such as Salmonella, Bacillus Calmette-Guérin, or Bordetella, and the other instead using commensal bacteria, such as lactobacilli or certain streptococci and staphylococci as vectors. Among viral vectors, vaccinia was initially the primary choice but has progressively been replaced by other poxviruses, such as canary poxvirus, and to an increasing extent by adenoviruses. Several of the live vectors of both bacterial and viral origins have also been used to provide various cytokines to further stimulate or modulate the immune responses induced. However, although many of these systems have shown promise and some have been used in clinical phase I or phase II studies, there is still no such vectored vaccine, whether inert or live, that has been approved for human use. ${ }^{59}$ The difficulty most often met by these approaches has been to achieve an acceptable level of attenuation while preserving the ability of the vector to express sufficient quantities of the desired antigen(s). Moreover, as has been clearly shown for adenoviruses, immunity to the vector may be so pronounced that it precludes reusing of the same vector for booster vaccinations.

The most promising results in recent years are from the use of so-called pseudoviruses or VLP. These are self-assembling nonreplicating viral core structures, often from nonenveloped viruses, which are produced recombinantly in vitro and even in plants. ${ }^{60-63}$ VLP are cheap and easy to make, as well as highly immunogenic, and are therefore of commercial interest as viral vaccines in their own right, with the recently licensed HPV vaccines being the primary success case. VLP can also be used as carriers/adjuvants both for foreign antigens expressed recombinantly on the surface of the pseudovirus, and for DNA 
vaccines carried within VLP. VLPs are especially interesting from a mucosal vaccine point of view as they offer opportunity to use the natural route of transmission of the parent virus for vaccine delivery. A promising use of this principle, resulting in both S-IgA and cytotoxic T-cell mucosal immune responses and protection against mucosal pathogen challenge, has been reported from studies both in animals and in humans with VLP from several mucosal viral pathogens including not only papilloma- but also noro- and hepatitis E-viruses. ${ }^{64}$

\section{Topical mucosal adjuvants}

Many types of adjuvants have been developed for topical, especially mucosal, administration, as exemplified in Table 4. These include bacterial enterotoxins, such as $\mathrm{CT}$ and its closely related E. coli heat-labile toxin (LT) analog, various Toll-like receptor ligands, interleukin 1 (IL-1) and IL-1 inducers, synthetic mast cell activators, retinoic acid, and other vitaminbased adjuvants.

The best-studied and most potent mucosal adjuvants in experimental systems are CT and LT. ${ }^{65-67}$ Besides being strong mucosal immunogens, both molecules are powerful mucosal adjuvants. They strongly potentiate the immunogenicity of most other antigens, whether these are linked to or simply admixed with the toxins, provided that the other antigen is given at the same time and at the same mucosal surface as the toxins. CT and LT can affect several steps in the induction of a mucosal immune response, which alone or in combination might explain their strong adjuvant action after oral immunization. Thus, CT has been found to (i) induce increased permeability of the intestinal epithelium leading to enhanced uptake of coadministered antigens; (ii) induce enhanced antigen presentation by various APCs; (iii) promote isotype differentiation in B cells leading to increased IgA formation; and (iv) exert complex stimulatory and inhibitory effects on T-cell proliferation and cytokine production. Related to this, both CT and LT have been shown to not only avoid to induce oral tolerance but also to abrogate otherwise efficient regimens for tolerance induction by oral antigen administration.

Among these many effects, those leading to enhanced antigen presentation by various APCs are probably of the greatest importance for the adjuvant activity. CT or LT markedly increase antigen presentation by DCs, macrophages, and B cells. ${ }^{66}$ They have also been found, at least in vitro, to stimulate intestinal epithelial cells to become effective APCs. Consistent with this activity, CT/LT upregulates the expression of MHC/HLA-DR molecules, CD80 and CD86 costimulatory molecules, as well as chemokine receptors such as CCR7 and CXCR4 on both murine and human DCs and other APCs. Importantly, CT/LT also induces the secretion of interleukin- $1 \beta$ from both DCs and macrophages. Interleukin-1 not only induces the maturation of DCs, but is also by itself an efficient mucosal adjuvant when coadministered with protein antigens and might mediate a significant part of the adjuvant activity of CT. ${ }^{68}$

In their own right both $\mathrm{CT}$ and $\mathrm{LT}$ in general are too toxic for being used as adjuvants for human vaccines. When given by oral administration to human volunteers, already $5 \mu \mathrm{g}$ of CT
Table 4 Mucosal adjuvants

\begin{tabular}{|c|c|}
\hline Enterotoxin-based adjuvants & $\begin{array}{l}\text { Cholera toxin (CT) and E. coli } \\
\text { heat-labile toxin (LT) } \\
\text { Detoxified CT and LT derivatives } \\
\text { CTA derivatives }\end{array}$ \\
\hline TLR ligands & $\begin{array}{l}\text { MPL (TLR2 and TLR4 ligands) } \\
\text { CpG ODN (TLR9 ligand); } \\
\text { CTB-CpG } \\
\text { Flagellin (TLR5 ligand) }\end{array}$ \\
\hline Nano- and micro-particulate & $\begin{array}{l}\text { ISCOMs, liposomes, } \\
\text { poly(DL-lactide-co-glycolide) } \\
\text { microspheres, cochleates, } \\
\text { polyphosphazenes }\end{array}$ \\
\hline $\begin{array}{l}\text { Low-molecular-weight } \\
\text { natural adjuvants }\end{array}$ & $\begin{array}{l}\text { IL-1 } \\
\text { Mast cell activators } \\
\text { Vitamins: } A \text { (retinoic acid), } D_{3}, E\end{array}$ \\
\hline
\end{tabular}

Abbreviations: IL, interleukin; ISCOMs, immune-stimulating complexes; MPL, monophosphoryl lipid A; TLR, Toll-like receptor.

together with bicarbonate buffer gave rise to profuse diarrhea, and when an influenza vaccine was adjuvanted with $2 \mu \mathrm{g}$ of LT and given intranasally, it was associated with Bell's palsy in a number of vaccinees leading to the rapid withdrawal of this vaccine from the market. ${ }^{69}$ Similar side effects have recently been reported after nasal administration of an enzymatically inactive LT mutant, ${ }^{70}$ suggesting that ADP ribosyl transferase activity and neurotoxicity are not linked. However, there appears to exist one exception to this rule inasmuch as LT has been used without reported side effects on the skin by transcutanous patch application also in humans both as an immunogen to stimulate antitoxic immunity against traveller's diarrhea, and as an adjuvant to stimulate an enhanced immune response to a coadministered vaccine antigen. ${ }^{44,45}$

To avoid the toxicity problems with whole CT or LT, especially for mucosal application, the recombinantly produced CTB and LTB proteins have been explored for their ability to increase immune responses against coadministered antigens. However, their capacity as mucosal adjuvants has proved to be much less than that of the holotoxins. Thus, both CTB and LTB are poor adjuvants when given to animals together with noncoupled antigens by the oral route, although they display a significant adjuvant activity when administered via the nasal route. Adjuvanticity of CTB or LTB is much improved when coupled to antigens. This is both due to the increased uptake of the coupled antigen across the mucosal barrier and the more efficient GM1 receptor-mediated uptake and presentation of the coupled antigen by APCs, including DCs and macrophages, as well as B cells.

Another approach to overcome the toxicity problem while still retaining significant adjuvant activity has been through various molecular engineering approaches illustrated in Figure 2. By mutations of specific residues involved in either the active site of the A subunit or in residues being critical for proteolytic release of the toxic-active A1 moiety from the A2 part associating with the $\mathrm{B}$-subunit pentamer, various LT and CT A-subunit mutants have been generated, which are substantially reduced in, or in some cases practically devoid of, enterotoxic activity, but which 
1. Single mutations in ADPR site, e.g., LTK63

2. Single or double mutations to block "nicking," e.g., LT-RG192 and LT- R192G/L211A (dmLT)

3. Peptide extension to block ADPR site e.g., 23-CT

4. Coupling CTA1 to APC-binding protein, CTA1-DD

5. Coupling TLR agonist to CTB, CTB-CpG

6. CTA1 with cell-penetration peptide, T-CTA1-T

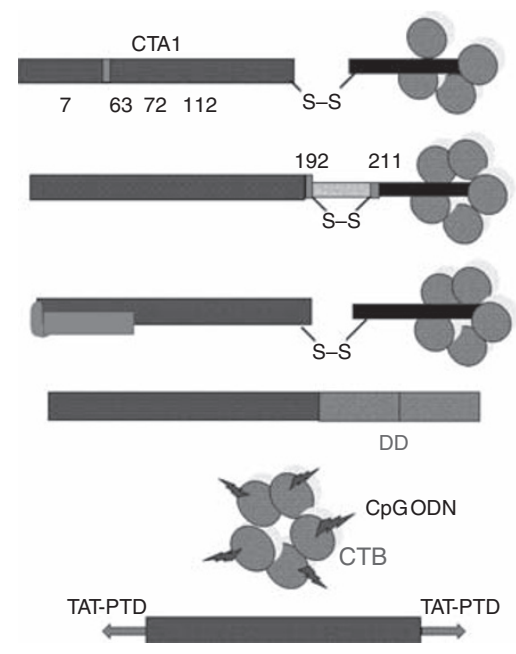

Figure 2 Examples of topical adjuvants derived from cholera toxin (CT) and E. coli heat-labile enterotoxin (LT) developed for mucosal and transcutaneous immunization. ADPR, ADP ribosyl transferase; APC, antigen-presenting cell; CTB, cholera toxin B subunit; TLR, Toll-like receptor.

retain significant adjuvant activity when given to animals by a mucosal route. A few of these adjuvants, such as LTK63 and LT-RG192, have been tested in humans, LTK63 together with influenza vaccine by nasal spray administration and LT-RG192 together with a killed Campylobacter whole-cell vaccine; in both cases, a significant adjuvant effect was found. The LT-RG192adjuvanted vaccine, however, gave diarrheal side effects in some individuals, and in two follow-up studies of different other vaccines given intranasally together with LTK63, there have been a few observations of Bell's palsy after vaccination. The probably most promising of the different toxin-based adjuvants in these categories at the present time is the double-mutated LT R192G/L211A (dmLT) developed by JD Clements et al. (to be published), which, in preclinical studies, has been devoid of practically all toxicity and yet retained strong adjuvant activity both orally and when given by sublingual administration; the dmLT protein is currently being tested for adverse reactions in a phase I study in US volunteers and if found adequately nontoxic planned to be tested in combination with an oral vaccine against ETEC.

An alternative approach has been taken by Agren et al., ${ }^{70}$ who instead of attenuating the A subunit have made a gene fusion protein between fully active CTA1 and a Staphylococcus aureus protein-A derivative named DD (Figure 2). The CTA1DD fusion protein binds specifically to immunoglobulins on antigen-presenting $\mathrm{B}$ cells via the DD protein and induces ADP ribosylation by the CTA1 moiety. When given intranasally together with protein antigens, CTA1-DD substantially increases both mucosal and systemic immune responses.

Yet another type of promising adjuvant protein was recently described by Adamsson et al. ${ }^{72}$ They coupled a well-known CpG oligonucleotide adjuvant to CTB (Figure 2) and showed that the CpG-CTB conjugate had markedly increased activity in activating different APCs in vitro and in stimulating both T-cell and antibody responses in vivo. ${ }^{72}$

Finally, even the CTA1 molecule itself, especially when decorated at both the amino and the carboxy ends with a cell penetration-enhancing peptide (the protein transduction domains from human immunodeficiency virus type 1 Tat protein) (Figure 2), has shown promising adjuvant activity when given sublingually together with influenza vaccine (M Song et al., to be published).

\section{“Tropical barriers" to topical vaccines}

The oral polio vaccine (OPV), which was licensed more than 50 years ago, is a classic oral mucosal vaccine. In addition to its enormous impact in eradicating polio in most countries, this vaccine has served as a useful tool in elucidating the fundamental aspects of mucosal immunity in humans. ${ }^{2,4,28,30}$ Similar to the injectable, inactivated polio vaccine (IPV), but five times cheaper, OPV produces antibodies in the blood that prevent dissemination of poliovirus to the nervous system. However, unlike IPV, OPV also produces a local S-IgA immune response in the intestinal mucosa-the primary site for poliovirus entry. This intestinal immune response is the most critical property of the OPV as it can rapidly stop personto-person transmission of wild poliovirus ("herd protection"), making mass campaigns with OPV a most powerful strategy for the global eradication of polio. This would not be feasible with the injectable IPV. However, at the same time, concerns have been raised after reports have shown low to no response to OPV in children from certain developing countries, even after as many as $10 \mathrm{OPV}$ doses were given. Similar to the OPV situation, several live oral vaccines have performed poorly in developing countries compared with industrialized countries. This is attributed mainly to chronic environmental enteropathy (CEE), also called tropical enteropathy, which is characterized by disturbances of digestive and absorptive functions. Factors that may contribute to CEE include poor sanitation, intestinal flora overgrowth, and histological changes, characterized by inflammation and blunting of small intestinal villi leading to malabsorption. ${ }^{73-76}$ Children living under extreme poverty are especially sensitive to this condition. 
Other factors that might hurt the performance of oral vaccines in developing countries include deficiencies in nutrients such as vitamin A (retinoic acid) and zinc, which can influence the response to oral adjuvants and vaccines by affecting discrete subpopulations of intestinal DCs and T cells; persistant activation of the gut innate immune system by infectious agents, such as intestinal helminths and flagellated protozoan parasites (especially Giardia) and concomitant viral and bacterial infections; and interferences by maternal breast milk (breast milk from mothers of low socioeconomic status in developing countries contains high titers of antibodies to enteric pathogens that can interfere with oral vaccine "take"). Strategies for coping with the different causes of immune hyporesponsiveness to oral vaccines in developing countries include the coadministration of vaccines with agents that improve gut integrity, such as zinc, vitamin $\mathrm{A}$, and possibly probiotics; withdrawal of breast milk shortly before oral vaccination; and treatment of parasite infestation before oral immunization. It would also be interesting to find whether vaccines administered by a nonintestinal route (e.g., nasal, sublingual, transcutaneous) could overcome these gut barriers.

\section{Surrogates of mucosal vaccine-induced immunity}

As of today, there is no method that has been qualified by regulatory bodies to measure mucosal immune responses to vaccines. Traditional approaches such as measurement of secretory antibodies in external secretions (saliva, tears, nasal, and genital secretions) and in organ lavages (gut, bronchoalveolar) using sero-immunoassays have not gained general acceptance, having either met with problems of reproducibility (even for a given individual tested on several occasions in a single day) or with practical problems linked to their inability to perform on a large scale (e.g., gut and bronchoalveolar lavages), limiting use of these methods, especially in young infants and children.

Probably the most challenging problem that these methods have met and will continue to face is the inherent compartmentalization of immune responses induced by mucosal immunization. Thus, immunity expressed and measured in secretions from a given organ (e.g., saliva) may not faithfully reflect immunity induced in the target organ (e.g., genital tract, large intestine).

Several approaches are now being developed largely based on improved knowledge of mechanisms governing dissemination of mucosal immune responses and especially of mucosal plasmablasts. One such approach utilizes the known ability of recently activated antibody-secreting plasmablasts to circulate in blood after antigen/vaccine exposure, regardless of where these cells had been activated. Combined immunomagnetic cell-sorting and enzyme-linked immunospot assays can now be performed on small samples of whole blood (without previous gradient enrichment of mononuclear cells) and allows for partitioned measurement of systemic and mucosal antibody responses to vaccines by detecting antigen-specific plasmablasts with a specific mucosal pedigree (e.g., $\alpha 4 \beta 7$, CCR10). This approach may in the future be expanded to cells with defined mucosal tissue tropism, such as cells expressing markers specifying receptors for small intestine, large intestine, lung, and genital tract tissues.

\section{PERSPECTIVES}

Better knowledge of human mucosal immune responsiveness during early life is required to establish the usefulness of different routes of vaccine administration against pathogens encountered by neonates and young infants from developing and industrialized countries.

To explore the impact of environmental factors (tropical enteropathy, malnutrition, and maternal factors) in relation to mucosal responses to vaccines administered by different routes, studies should be conducted with licensed vaccines, both killed and live bacterial and viral vaccines, and for comparison, also with live and killed parenteral vaccines (e.g., OPV and IPV) in developing countries. Use of animal models could also be helpful in exploring the influence of these factors on mucosal immune responsiveness to antigens and adjuvants administered by these different routes.

The choice of any given route of mucosal vaccination will affect vaccine design, and selection of appropriate adjuvants and formulations, and thus it will influence vaccine process and manufacturing issues. Compared with most licensed injectable vaccines, it is interesting to note that currently, there are no pure subunit vaccines formulated and licensed for mucosal administration. As most injectable subunit vaccines are given with an adjuvant, a further challenge in the field will likely be the development of adjuvants that enhance the potency of future subunit vaccines administered by different mucosal routes.

\section{ACKNOWLEDGMENTS}

We express our gratitude to our collaborators, colleagues, and students for their contributions to the studies summarized here. The International Vaccine Institute is supported by the governments of the Republic of Korea, Sweden (SIDA), the Netherlands, and Kuwait.

\section{DISCLOSURE}

The authors declared no conflict of interest.

(C) 2010 Society for Mucosal Immunology

\section{REFERENCES}

1. Brandtzaeg, P. \& Pabst, R. Let's go mucosal: communication on slippery ground. Trends Immunol. 25, 570-577 (2004).

2. Ogra, PL. Developmental aspects of the mucosal immune system: role of external environment, mucosal microflora and milk. Adv. Exp. Med. Biol. 639, 41-56 (2009).

3. Holmgren, J. \& Czerkinsky, C. Mucosal immunity and vaccines. Nat. Med. 11, S45-S53 (2005).

4. Ogra, P.L. \& Karzon, D.T. Distribution of poliovirus antibody in serum, nasopharynx and alimentary tract following segmental immunization of lower alimentary tract with poliovaccine. J. Immunol. 102, 1423-1430 (1969).

5. Kiyono, H. \& Fukuyama, S. NALT- vs. Peyer's-patch-mediated mucosal immunity. Nat. Rev. Immunol. 4, 699-710 (2004).

6. Mestecky, J. et al. Oral immunization: an update. Curr. Opin. Gastroenterol. 24, 713-719 (2008).

7. Kraehenbuhl, J.P. \& Neutra, M.R. Epithelial M cells: differentiation and function. Annu. Rev. Cell Dev. Biol. 16, 301-332 (2000).

8. Jang, M.H. et al. Intestinal villous M cells: an antigen entry site in the mucosal epithelium. Proc. Natl. Acad. Sci. USA 101, 6110-6115 (2004).

9. Rescigno, M. \& Di Sabatino, A. Dendritic cells in intestinal homeostasis and disease. J. Clin. Invest. 119, 2441-2450 (2009). 
10. Mowat, A.M. Anatomical basis of tolerance and immunity to intestinal antigens. Nat. Rev. Immunol. 3, 331-341 (2003).

11. Rakoff-Nahoum, S. et al. Recognition of commensal microflora by toll-like receptors is required for intestinal homeostasis. Cell 118, 229-241 (2004).

12. Bilsborough, J. \& Viney, J.L. Gastrointestinal dendritic cells play a role in immunity, tolerance, and disease. Gastroenterology 127, 300-309 (2004).

13. Fahlen-Yrlid, L. et al. CD11c(high) dendritic cells are essential for activation of CD4+ T cells and generation of specific antibodies following mucosal immunization. J. Immunol. 183, 5032-5041 (2009).

14. Berg, E.L. et al. Homing receptors and vascular addressins: cell adhesion molecules that direct lymphocyte traffic. Immunol. Rev. 108, 5-18 (1989).

15. Kunkel, E.J. \& Butcher, E.C. Plasma-cell homing. Nat. Rev. Immunol. 3, 822-829 (2003).

16. Mora, J.R. et al. Generation of gut-homing IgA-secreting B cells by intestinal dendritic cells. Science 314, 1157-1160 (2006).

17. Stagg, A.J. et al. Intestinal dendritic cells increase T cell expression of alpha4beta7 integrin. Eur. J. Immunol. 32, 1445-1454 (2002).

18. Mora, J.R. et al. Selective imprinting of gut-homing T cells by Peyer's patch dendritic cells. Nature 424, 88-93 (2003).

19. Johansson-Lindbom, B. et al. Functional specialization of gut CD103+ dendritic cells in the regulation of tissue-selective T cell homing. J. Exp. Med. 202, 1063-1073 (2005).

20. Iwasaki, A. Mucosal dendritic cells. Annu. Rev. Immunol. 25, 381-418 (2007).

21. Wilson, E. \& Butcher, E.C. CCL28 controls immunoglobulin (lg)A plasma cell accumulation in the lactating mammary gland and IgA antibody transfer to the neonate. J. Exp. Med. 200, 805-809 (2004).

22. Czerkinsky, C. et al. Antibody-producing cells in peripheral blood and salivary glands after oral cholera vaccination of humans. Infect. Immun. 59, 996-1001 (1991).

23. Eriksson, K. et al. Specific-antibody-secreting cells in the rectums and genital tracts of non-human primates following vaccination. Infect. Immun. 66, 5889-5896 (1998).

24. Jertborn, M. et al. Local and systemic immune responses to rectal administration of recombinant cholera toxin B subunit in humans. Infect. Immun. 69, 4125-4128 (2001).

25. Johansson, E.L. et al. Comparison of different routes of vaccination for eliciting antibody responses in the human stomach. Vaccine 22, 984-990 (2004).

26. Kozlowski, P.A. et al. Comparison of the oral, rectal, and vaginal immunization routes for induction of antibodies in rectal and genital tract secretions of women. Infect. Immun. 65, 1387-1394 (1997).

27. Quiding, M. et al. Intestinal immune responses in humans. Oral cholera vaccination induces strong intestinal antibody responses and interferongamma production and evokes local immunological memory. J. Clin. Invest. 88, 143-148 (1991).

28. Ogra, P.L. \& Ogra, S.S. Local antibody response to poliovaccine in the human female genital tract. J Immunol. 110, 1307-1311 (1973).

29. Wassén, L. et al Local intravaginal vaccination of the female genital tract. Scand. J. Immunol. 44, 408-414 (1996).

30. Ogra, P.L. \& Karzon, D.T. Poliovirus antibody response in serum and nasal secretions following intranasal inoculation with inactivated poliovaccine. J. Immunol. 102, 15-23 (1969).

31. Quiding-Järbrink, M. et al. Differential expression of tissue-specific adhesion molecules on human circulating antibody-forming cells after systemic, enteric, and nasal immunizations. A molecular basis for the compartmentalization of effector B cell responses. J. Clin. Invest. 99, 1281-1286 (1997).

32. Quiding-Järbrink, M. et al. Induction of compartmentalized B-cell responses in human tonsils. Infect. Immun. 63, 853-857 (1995).

33. Rudin, A. et al. Differential kinetics and distribution of antibodies in serum and nasal and vaginal secretions after nasal and oral vaccination of humans. Infect. Immun. 66, 3390-3396 (1998).

34. Johansson, E.L. et al. Nasal and vaginal vaccinations have differential effects on antibody responses in vaginal and cervical secretions in humans. Infect. Immun. 69, 7481-7486 (2001).

35. Jertborn, M. et al. Immunological memory after immunization with oral cholera B subunit-whole-cell vaccine in Swedish volunteers. Vaccine 12 1078-1082 (1994)

36. Cuburu, N. et al. Sublingual immunization induces broad-based systemic and mucosal immune responses in mice. Vaccine 25, 8598-8610 (2007).
37. Song, J.H. et al. Sublingual vaccination with influenza virus protects mice against lethal viral infection. Proc. Natl. Acad. Sci. USA 105, 1644-1649 (2008).

38. Cuburu, N. et al. Sublingual immunization with nonreplicating antigens induces antibody-forming cells and cytotoxic T cells in the female genital tract mucosa and protects against genital papillomavirus infection. J. Immunol. 183, 7851-7859 (2009).

39. Raghavan S. et al. Sublingual immunization protects against Helicobacter pylori infection and induces $\mathrm{T}$ and $\mathrm{B}$ cell responses in the stomach. Infect. Immun. in press (2010).

40. Song, J.H. et al. CCR7-CCL19/CCL21-regulated dendritic cells are responsible for effectiveness of sublingual vaccination. J. Immunol. 182, 6851-6860 (2009).

41. Wang, W. et al. Identification of a novel CC chemokine (CCL28) which binds CCR10 (GPR2). J. Biol. Chem. 275, 22313-22323 (2000).

42. Pan, J. et al. A novel chemokine ligand for CCR10 and CCR3 expressed by epithelial cells in mucosal tissues. J. Immunol. 165, 2943-2949 (2000).

43. Kunkel, E.J. et al. CCR10 expression is a common feature of circulating and mucosal epithelial tissue IgA Ab-secreting cells. J. Clin. Invest. 111, 1001-1010 (2003).

44. Glenn, G.M. et al. Skin immunization made possible by cholera toxin. Nature 391, 851 (1998).

45. Glenn, G.M. \& Kenney, R.T. Mass vaccination: solutions in the skin. Curr. Top. Microbiol. Immunol. 304, 247-268 (2006).

46. Chang, S.Y. et al. Cutting edge: Langerin+ dendritic cells in the mesenteric lymph node set the stage for skin and gut immune system cross-talk. J. Immunol. 180, 4361-4365 (2008).

47. Herremans, T.M., Reimerink, J.H., Buisman, A.M., Kimman, T.G. \& Koopmans, M.P. Induction of mucosal immunity by inactivated poliovirus vaccine is dependent on previous mucosal contact with live virus. J. Immunol. 162, 5011-5018 (1999).

48. Svennerholm, A.M. et al. Boosting of secretory lgA antibody responses in man by parenteral cholera vaccination. Scand. J. Immunol. 6, 1345-1349 (1977).

49. Lavelle, E.C. \& O'Hagan, D.T. Delivery systems and adjuvants for oral vaccines. Expert Opin. Drug. Deliv. 3, 747-762 (2006).

50. Cox, E. et al. Adjuvants modulating mucosal immune responses or directing systemic responses towards the mucosa. Vet. Res . 37, 511-539 (2006).

51. Moyle, P.M. et al. Mucosal immunization: adjuvants and delivery systems. Curr. Drug. Deliv. 1, 385-396 (2004).

52. Nordly, P. et al. Status and future prospects of lipid-based particulate delivery systems as vaccine adjuvants and their combination with immunostimulators. Expert Opin. Drug Deliv. 6, 657-672 (2009).

53. Kersten, G.F. \& Crommelin, D.J. Liposomes and ISCOMs. Vaccine 21, 915-920 (2003).

54. Rao, R., Squillante, E. III. \& Kim, K.H. Lipid-based cochleates: a promising formulation platform for oral and parenteral delivery of therapeutic agents. Crit. Rev. Ther. Drug Carrier Syst. 24, 41-61 (2007).

55. McDermott, M.R. et al. Polymer-grafted starch microparticles for oral and nasal immunization. Immunol. Cell Biol. 76, 256-262 (1998).

56. Eldridge, J.H. et al. Biodegradable microspheres: vaccine delivery system for oral immunization. Curr. Top. Microbiol. Immunol. 146, 59-66 (1989).

57. Payne, L.G. et al. Water-soluble phosphazene polymers for parenteral and mucosal vaccine delivery. Pharm. Biotechnol. 6, 473-493 (1995).

58. Payne, L.G. et al. Poly[di(carboxylatophenoxy)phosphazene] (PCPP) is a potent immunoadjuvant for an influenza vaccine. Vaccine 16, 92-98 (1998).

59. Ada, G. Overview of vaccines and vaccination. Mol. Biotechnol. 29, 255-272 (2005).

60. Schiller, J.T. \& Lowy, D.R. Vaccines to prevent infections by oncoviruses. Annu. Rev. Microbiol. in press (2010).

61. Treanor, J. Recombinant proteins produced in insect cells. Curr. Top. Microbiol. Immunol. 333, 211-225 (2009).

62. D'Aoust, M.A. et al. The production of hemagglutinin-based virus-like particles in plants: a rapid, efficient and safe response to pandemic influenza. Plant Biotechnol. J. 8, 607-619 (2010).

63. Ludwig, C. \& Wagner, R. Virus-like particles-universal molecular toolboxes. Curr. Opin. Biotechnol. 18, 537-545 (2007).

64. Jennings, G.T. \& Bachmann, M.F. The coming of age of virus-like particle vaccines. Biol. Chem. 389, 521-536 (2008).

65. Freytag, L.C. \& Clements, J.D. Mucosal adjuvants. Vaccine 23, 1804-1813 (2005). 
66. Holmgren, J. et al. Mucosal adjuvants and anti-infection and antiimmunopathology vaccines based on cholera toxin, cholera toxin B subunit and CpG DNA. Immunol. Lett. 97, 181-188 (2005).

67. Lycke, N. ADP-ribosylating bacterial enzymes for the targeted control of mucosal tolerance and immunity. Ann. NY Acad. Sci.. 1029, 193-208 (2004).

68. Tagliabue, A. \& Boraschi, D. Cytokines as vaccine adjuvants: interleukin 1 and its synthetic peptide 163-171. Vaccine 11, 594-615 (1993).

69. Couch, R.B. Nasal vaccination, Escherichiacoli enterotoxin, and Bell's palsy. N. Engl. J. Med. 350, 860-861 (2004).

70. Lewis, D.J. et al. Transient facial nerve paralysis (Bell's palsy) following intranasal delivery of a genetically detoxified mutant of Escherichiacoli heat labile toxin. PLoS One. 4, e6999 (2009).
71. Agren, L.C. et al. Genetically engineered nontoxic vaccine adjuvant that combines $B$ cell targeting with immunomodulation by cholera toxin A1 subunit. J. Immunol. 158, 3936-3946 (1997).

72. Adamsson, J. et al. Novel immunostimulatory agent based on CpG DNA linked to the non-toxic B subunit of cholera toxin. J. Immunol. 176, 4902-4913 (2006).

73. Mathan, V.I. Tropical sprue in southern India. Trans. R. Soc. Trop. Med. Hyg. 82, 10-14 (1988).

74. Nath, S.K. Tropical sprue. Curr. Gastroenterol. Rep. 7, 343-349 (2007).

75. Owens, S.R. \& Greenson, J.K. The pathology of malabsorption: current concepts. Histopathology 50, 64-82 (2007).

76. Humphrey, J.H. Child undernutrition, tropical enteropathy, toilets, and handwashing. Lancet 374, 1032-1035 (2009). 